\title{
The Highly Conserved COPII Coat Complex Sorts Cargo from the Endoplasmic Reticulum and Targets It to the Golgi
}

\author{
Christopher Lord ${ }^{1}$, Susan Ferro-Novick ${ }^{1}$, and Elizabeth A. Miller ${ }^{2}$ \\ ${ }^{1}$ Department of Cellular and Molecular Medicine, Howard Hughes Medical Institute, University of \\ California at San Diego, La Jolla, California 92093 \\ ${ }^{2}$ Department of Biological Sciences, Columbia University, New York, New York 10027 \\ Correspondence: em2282@columbia.edu; sfnovick@ucsd.edu
}

Protein egress from the endoplasmic reticulum (ER) is driven by a conserved cytoplasmic coat complex called the COPII coat. The COPII coat complex contains an inner shell (Sec23/ Sec24) that sorts cargo into ER-derived vesicles and an outer cage (Sec13/Sec31) that leads to coat polymerization. Once released from the ER, vesicles must tether to and fuse with the target membrane to deliver their protein and lipid contents. This delivery step also depends on the COPII coat, with coat proteins binding directly to tethering and regulatory factors. Recent findings have yielded new insight into how COPII-mediated vesicle traffic is regulated. Here we discuss the molecular basis of COPII-mediated ER-Golgi traffic, focusing on the surprising complexity of how ER-derived vesicles form, package diverse cargoes, and correctly target these cargoes to their destination.

\begin{abstract}
The port of entry into the secretory pathway is the endoplasmic reticulum (ER). Approximately one-third of the eukaryotic proteome traffics from this multifunctional organelle (Huh et al. 2003). This diverse set of cargo is translocated into the ER, folded, and modified before it travels to the Golgi, where further modifications occur. From the Golgi, cargo is sorted to other subcellular compartments to perform a variety of cellular functions. The highly conserved machinery required for these transport events was initially identified through genetic screens in the yeast Saccharomyces cerevisiae,
\end{abstract}

and insights into the function of this machinery were provided through the use of in vitro transport assays. Advances in microscopy, in particular, the use of GFP fusion proteins and live cell imaging, have also played a critical role in understanding the dynamics of membrane traffic. In this article, we describe the mechanistic advances that have helped us to understand how diverse cargo correctly traffics from the ER to the Golgi complex in lower and higher eukaryotes. Even though these mechanisms are largely conserved, they are more complex at the molecular and organizational levels in metazoans.

Editors: Susan Ferro-Novick, Tom A. Rapoport, and Randy Schekman

Additional Perspectives on The Endoplasmic Reticulum available at www.cshperspectives.org

Copyright (C) 2013 Cold Spring Harbor Laboratory Press; all rights reserved; doi: 10.1101/cshperspect.a013367

Cite this article as Cold Spring Harb Perspect Biol 2013;5:a013367 


\section{THE COPII COAT GENERATES ER-DERIVED VESICLES}

Our understanding of the molecular basis of vesicle-mediated ER export has been built on the isolation and characterization of conditional lethal yeast secretory mutants that accumulate ER membranes at their restrictive growth temperatures (Novick et al. 1980, 1981). This genetic approach, coupled with subsequent biochemical characterization of ER-Golgi transport events, ultimately defined the vesicle coat proteins themselves, known as the COPII coat (Baker et al. 1988; Ruohola et al. 1988; Barlowe et al. 1994). The five highly conserved cytosolic proteins that define the coat complex represent the minimal machinery required for generating and populating spherical transport vesicles from the ER membrane (Barlowe et al. 1994; Matsuoka et al. 1998a,b). On synthetic liposomes, the COPII coat assembles in a hierarchical manner, driven by the initial recruitment and activation of the small G-protein Sar1, which exists in a soluble, cytoplasmic form when in its GDP-bound state (Nakano and Muramatsu 1989; Barlowe et al. 1993). Exchange of GDP for GTP is catalyzed in vivo by the ER membrane protein Sec12, which activates Sar1 (Nakano et al. 1988; Barlowe and Schekman 1993). Activation of Sarl triggers a structural rearrangement that exposes an amphipathic $\alpha$-helix that shallowly embeds in the lipid bilayer and likely induces initial membrane curvature by locally expanding the cytoplasmic leaflet relative to the lumenal leaflet (Bielli et al. 2005; Lee et al. 2005). Membrane-bound GTP-loaded Sar1 subsequently recruits a heterodimer composed of Sec 23 and Sec24, which stably associate with each other to form a bowtie-shaped structure that exhibits a concave surface that may face the membrane (Matsuoka et al. 1998b; Bi et al. 2002). The Sec23/Sec24 complex provides two functions. Sec23 is the GTPase-activating protein for Sar1, contributing to GTP hydrolysis via an "arginine finger" that inserts into the GTPbinding pocket (Yoshihisa et al. 1993; Bi et al. 2002). Sec24 is the cargo-binding platform, interacting with multiple different ER export motifs via a variety of surface-localized domains
(Miller et al. 2002, 2003; Mossessova et al. 2003; Mancias and Goldberg 2008). Sec23/Sec24 also provide for the recruitment of the outer layer of the COPII coat, a heterotetramer formed by Sec13 and Sec31.

The Sec13/Sec31 complex has the intrinsic capacity to self-assemble into cage-like lattice structures that can adopt multiple different geometries (Stagg et al. 2006, 2008). Crystal structures of fragments of Sec31 complexed with Sec13 suggest that the COPII cage assembles largely by intermolecular interactions between Sec31 molecules. Sec31 dimerizes tail-to-tail in the middle of the rod-shaped "edge" element via carboxy-terminal $\alpha$-solenoid domains; four amino-terminal $\beta$-barrel domains of Sec31 interact to drive assembly of the "vertex" region where four rods come together; and sandwiched between these two domains lies Sec13. In addition to providing a membrane-bending force, Sec31 also contributes to GTP hydrolysis via an unstructured loop within the proline-rich domain that lies across the surface of Sec23/ Sar1 to optimize the position of catalytic residues (Bi et al. 2007) and thereby stimulate the GTPase activity of the coat approximately 10fold (Antonny et al. 2001). Mammalian Sec31 is ubiquitinated, an event that is critical for efficient collagen traffic (Jin et al. 2012). ER export of collagen represents a unique challenge in that assembled collagen rods are too large to fit into a canonical COPII vesicle. It is tempting to speculate that ubiquitination may facilitate recruitment of an accessory factor that would change the geometry of the COPII cage or alter the rigidity of the coat to capture this large, unwieldy cargo more effectively.

Vesicle formation can be separated into three distinct events. First, membrane curvature is required to transform a planar ER membrane into a curved vesicle $(60-80 \mathrm{~nm}$ in vitro). Second, the capture of appropriate cargo molecules is needed to ensure efficient ER egress of key proteins. Third, scission of the membrane drives vesicle release from the donor membrane. As described above, membrane curvature likely stems from multiple physical properties of each of the COPII coat proteins. Sar1 can drive tubulation of synthetic liposomes upon insertion 
of its amphipathic $\alpha$-helix (Bielli et al. 2005; Lee et al. 2005). But ectopic recruitment of a truncated form of Sar1 that lacks this domain still results in the formation of a spherical bud-like structure upon the addition of Sec23/Sec24 and Sec13/Sec31 (Lee et al. 2005). This observation suggests that additional membranebending capacity derives from the rest of the coat. The spherical structure of the Sec13/ Sec31 cage is suggestive of a central role for the outer coat in generating membrane curvature, either by direct scaffolding or by organizing and concentrating the curvature induced by the underlying inner coat proteins (Stagg et al. 2006, 2008). Recent biochemical and genetic dissection of the molecular function of Sec13 in yeast has further defined a structural role for Sec13/Sec31 in generating vesicles from cargorich membranes, which may represent a significant barrier to membrane curvature induced by the coat (Copic et al. 2012).

Unlike membrane curvature, which seems to be driven by multiple coat elements, cargo recruitment into nascent vesicles is driven largely by the Sec24 subunit. A role for Sec23/Sec24 was initially proposed when a minimal "prebudding" complex composed of Sar1, Sec23, and Sec24 was shown to interact with cargo proteins (Aridor et al. 1998; Kuehn et al. 1998) and the SNARE (soluble $N$-ethylmaleimide-sensitive factor attachment protein receptor) machinery that drives downstream membrane fusion (Springer and Schekman 1998). Although some evidence exists for a direct role for Sar1 in the capture of cargo molecules (Giraudo and Maccioni 2003; Tabata et al. 2009), the overwhelming genetic, biochemical, and structural data support a primary role for Sec24 in this process (Miller et al. 2002, 2003; Mossessova et al. 2003; Mancias and Goldberg 2007, 2008). Furthermore, most eukaryotes express multiple paralogs of Sec 24 that have distinct cargo-binding capacities, which likely serves to diversify the repertoire of cargo molecules recognized by the coat (Roberg et al. 1999; Shimoni et al. 2000; Miller et al. 2002; Mancias and Goldberg 2008). Again, the transport of large cargo proteins seems to require additional functionality, linked either to the coat itself or to accessory proteins that facilitate uptake. The yeast Sec24 paralog, Lst1, is required for efficient transport of the plasma membrane protein, Pma1, which forms a dodecamer of $\sim 1 \mathrm{MDa}$ (Roberg et al. 1999; Lee et al. 2002). Vesicles formed with Lst1 are intrinsically larger in size, suggesting that vesicle architecture is in part dependent on coat composition (Shimoni et al. 2000). Similarly, collagen requires an accessory factor, TANGO1, which binds both collagen (in the lumen) and Sec23/Sec24 (in the cytosol) and may alter vesicle architecture or regulate the coat to prevent premature vesicle scission (Saito et al. 2009; Malhotra and Erlmann 2011). cTAGE5, a TANGO1 homolog, binds both TANGO1 and Sec23/ $\operatorname{Sec} 24$ and is also required for collagen export from the ER (Fig. 1).

Once a nascent vesicle bud has formed and been adequately populated, the membrane at the bud neck must undergo scission to release the free vesicle and close the ER membrane. In other vesicle scission events, this step can be driven by members of the dynamin family, large GTPases that couple GTP hydrolysis to membrane-remodeling events. There is no evidence for a dynamin-like protein in ER export, and instead this function is thought to be driven by Sar1 itself, with the GTPase activity driving membrane remodeling via insertion and removal of the amphipathic $\alpha$-helix (Pucadyil and Schmid 2009). Indeed, in budding experiments that are driven either by a GTP-locked form of Sar1 (Bielli et al. 2005) or by a truncated form that lacks the $\alpha$ helix (Lee et al. 2005), vesicle buds form but are not released from the donor membrane, confirming a role for Sar1 in vesicle scission. However, it is becoming clear that additional layers of regulation broadly act on the process of vesicle formation and scission in particular. Recent biochemical dissection of the essential but enigmatic protein Sec16 uncovered a GTPase inhibitory function for a central domain that likely works by preventing the efficient recruitment of Sec31 to the Sar1/Sec23 complex, thereby reducing the GTPase activity of the coat in situ. Surprisingly, this function of Sec16 was impaired by a surface mutation on Sec24, and one effect of the increased GTPase activity of the coat in the context of the Sec24-m11 mutant was the release 
C. Lord et al.
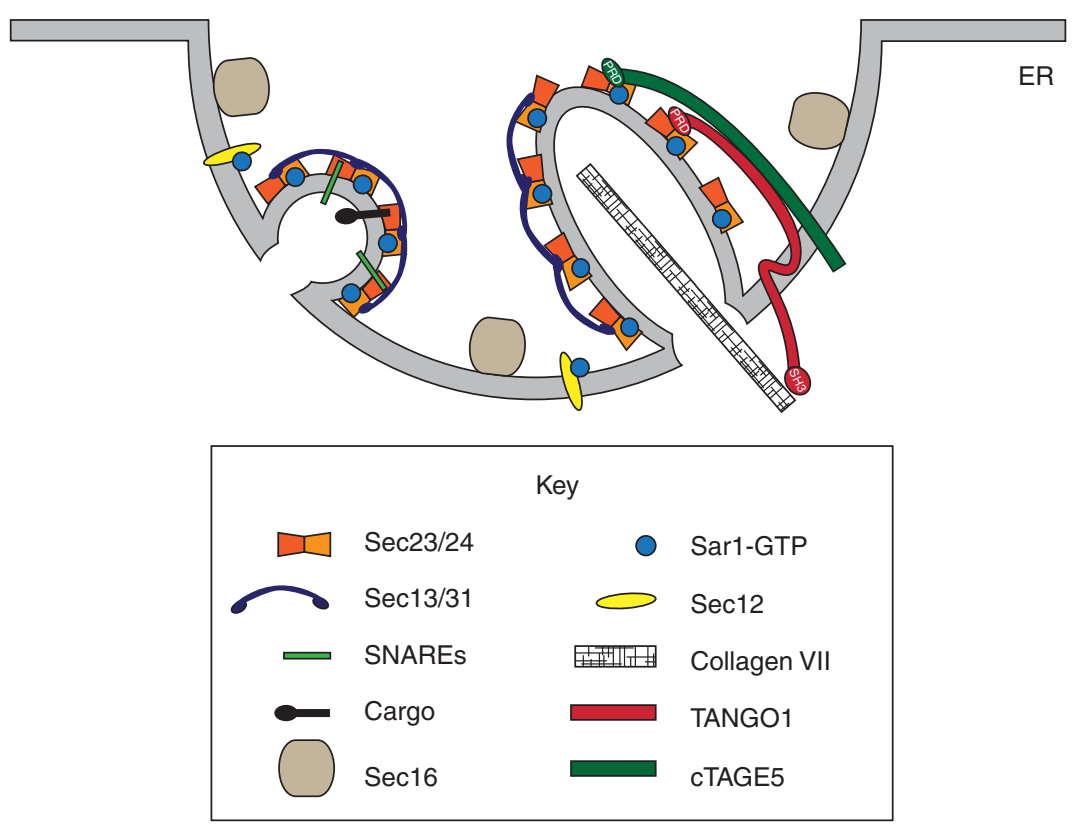

Figure 1. The COPII coat drives vesicle formation from the ER. COPII vesicle formation is initiated by the guanine nucleotide exchange factor Sec12, which recruits and activates Sar1. Sar1-GTP, in turn, recruits the Sec23/Sec24 dimer, which binds cargo molecules, including the SNARE proteins that drive vesicle fusion. These pre-budding complexes, in turn, recruit a heterotetramer of Sec13/Sec31, which drives membrane bending and vesicle formation. The peripheral protein Sec16 functions as both a regulator and a scaffold in this process, localizing to discrete ER exit sites that, in metazoan cells, form cup-shaped domains. Some large cargo proteins, like collagen, cannot fit into a canonical COPII vesicle but instead rely on accessory factors like TANGO1 and its homolog cTAGE5 to modify this process to generate a carrier competent for transport.

of smaller vesicles, confirming that GTPase activity of the coat is intimately linked to vesicle scission (Kung et al. 2012). These findings highlight the need for a deeper understanding of the complexity of intermolecular interactions that the coat proteins undergo in the complex environment of a cargo-rich membrane.

Although the fundamental machinery that drives COPII vesicle formation is conserved across all eukaryotes, metazoans have amplified the repertoire of COPII isoforms and also use additional accessory factors to modulate vesicle traffic in poorly understood ways. In some cases, this diversity likely reflects the specific challenges associated with the particular secretory needs of an individual cell. For example, collagen trafficking requires the accessory factors TANGO1 and cTAGE5, described above, but also seems to be particularly sensitive to perturbations in the level of Sec13/Sec31 (Townley et al. 2008). Similarly, uncoupling Sec13/Sec31 from the inner COPII coat by mutation of Sec23 causes a craniofacial development defect, presumably resulting from defects in collagen secretion (Fromme et al. 2007). This dependence on Sec13/Sec31 may reflect a need for significant membrane-bending force to encapsulate a collagen rod or may stem from altered GTPase activity of the coat under depletion conditions. As our understanding of the molecular basis of genetic disease increases, it is becoming increasingly apparent that COPII-mediated traffic can be linked to a growing number of different disease conditions. Some of these are cargo specific: Mutations in the cargo receptor ERGIC-53 cause blood clotting diseases as the result of inefficient ER export of clotting factors (Nichols and Ginsburg 1999). Others cause more broad 
cellular dysfunction: Sec16 interacts with a protein called TFG-1, which was originally identified as a gene fusion associated with thyroid cancer, and in this state drives recruitment of a kinase to ER exit sites, improperly phosphorylating unknown substrates and thereby causing a propensity for transformation (Witte et al. 2011).

\section{ER-TO-GOLGI TRAFFIC AND TWO COAT COMPLEXES}

In addition to the molecular regulation described above, ER export in both budding yeast and mammalian cells is subject to higher-level organization. Cargo exits the ER from a specialized domain where COPII vesicles form, known as the transitional ER or ER exit sites (Palade 1975). These domains serve in part to segregate different classes of cargo molecules (Muniz et al. 2001; Watanabe and Riezman 2004) and also participate in ER quality control by excluding misfolded proteins (Mezzacasa and Helenius 2002). Precisely how these discrete domains are generated and maintained remains unclear, although Sec16 seems to be a key player, perhaps via its scaffolding function (Ivan et al. 2008; Hughes et al. 2009). In mammalian cells, vesicles subsequently move to the pre-Golgi or ERGolgi intermediate compartment, called the ERGIC (also referred to as vesicular tubular clusters, or VTCs) (Fig. 2), which corresponds to the first post-ER compartment where sorting occurs (Bannykh et al. 1996). From the ERGIC, cargo either traffics toward the Golgi or back to the ER (Ben-Tekaya et al. 2005). The ERGIC resides near ER exit sites and has different properties than either the ER or Golgi (Schweizer et al. 1991). Early live cell imaging studies on the trafficking of the viral glycoprotein VSV-G suggested that the ERGIC might be a transient pre-Golgi intermediate that fuses with the cisGolgi compartment (Presley et al. 1997). A more recent study using GFP-ERGIC-53 as a marker, however, showed that ERGIC-53 localizes to stationary membranes that correspond to ERGIC clusters. GFP-ERGIC-53 did not display any net movement from these clusters, whereas the anterograde cargo marker VSV-G-GFP moved toward the Golgi. Cargo sorting from ERGIC-53-

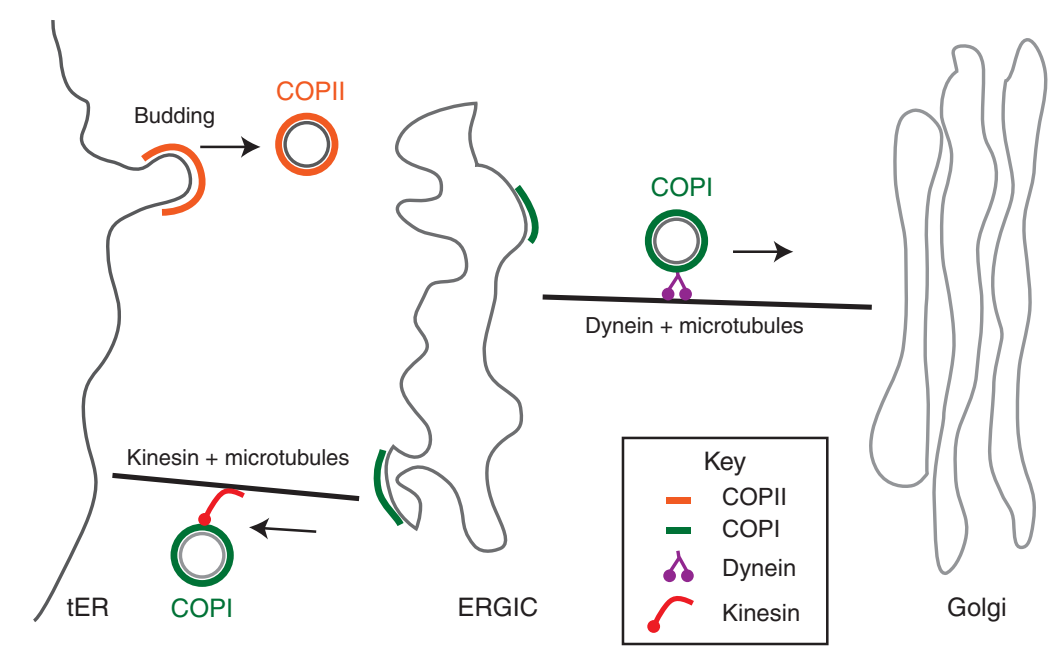

Figure 2. Vesicle traffic between the ER and the Golgi complex. Vesicle traffic from the ER is initiated when the COPII coat complex sorts cargo into a budding vesicle from the transitional ER (tER). Cargo then moves from the ER to the ER-Golgi intermediate compartment (ERGIC). This event does not require microtubules. Traffic from the ERGIC to the Golgi is dependent on microtubules and the motor protein dynein, and appears to be mediated by COPI-coated vesicles. Nonresident ERGIC cargo recycles back to the ER. Recycling is COPI dependent and requires microtubules, as well as the motor protein kinesin. 
C. Lord et al.

positive structures back to the ER was also observed, implying that ERGIC-53 marks a stable organelle (Ben-Tekaya et al. 2005).

The ERGIC has two main functions: to concentrate cargo that leaves the ER and to sort cargo for onward anterograde transport or retrograde retrieval back to the ER (Aridor et al. 1995; Martínez-Menárguez et al. 1999). Both of these cargo-sorting events are regulated by defined protein sequences. For example, a hydrophobic motif in GAT1, a transmembrane GABA transporter that traffics to the plasma membrane, regulates exit from the ERGIC toward the cis-Golgi (Farhan et al. 2008). The identification of a positive sorting signal to drive anterograde traffic from the ERGIC is consistent with the model of the ERGIC as a stable compartment, although the molecular mechanism by which such signals are recognized remains unknown. Retrograde traffic back to the ER is driven by different sorting sequences, such as KDEL (Munro and Pelham 1987) and dilysine (Jackson et al. 1990) motifs, which function either by direct interaction with the COPI coat (dilysine signals) or by indirect engagement via the KDEL receptor (Erd2 in yeast). COPI is a second coat complex that acts sequentially with the COPII complex on this pathway (Aridor et al. 1995). The COPI coat complex is recruited to the ERGIC to mediate anterograde traffic to the Golgi and retrograde traffic back to the ER (Pepperkok et al. 1993; Scales et al. 1997; Stephens and Pepperkok 2002). An in-depth discussion of the role of the COPI coat in retrograde traffic is discussed by Spang (2013).

Yeast appears to lack a distinct ERGIC, and, instead, the first post-ER compartment where sorting occurs is the early Golgi. Traffic from the ER to the Golgi is mechanistically similar to ER-ERGIC traffic, with COPII vesicles rapidly diffusing within the cell and fusing directly with the early Golgi (Cao et al. 1998; Lord et al. 2011). In the absence of an ERGIC compartment, COPI-mediated retrograde traffic in yeast is also from the early Golgi (Letourneur et al. 1994).

Because cargo selection and fusion factors need to recycle back to the ER to regulate additional anterograde cycles, disrupting retrograde transport typically affects anterograde traffic. One important unanswered question is how the same COPI coat complex can be used for different trafficking events within the ER-toGolgi pathway. Specificity may be regulated by different GTPases of the Arf1 family, which mediate the recruitment of the COPI coat complex to membranes (D'Souza-Schorey and Chavrier 2006; Hsu et al. 2009), or potentially by different types of cargo and their sorting motifs.

\section{THE ROLE OF THE COPII COAT IN CYTOSKELETAL-MEDIATED CARGO TRANSPORT}

Disruption of microtubules by the depolymerizing agent nocodazole inhibits the transport of VSV-G from the ERGIC to the Golgi but has no effect on the transport of VSV-G into the ERGIC (Presley et al. 1997). The large physical distance between the ERGIC and the Golgi may require microtubule function for the efficient transport of cargo between these two compartments (Fig. 2). Microtubule-driven traffic from the ERGIC to the Golgi also requires the minus-end-directed motor dynein and its adaptor complex dynactin, which links dynein to membranes (Presley et al. 1997; Kardon and Vale 2009). Additionally, the COPII coat subunit Sec23 interacts with the dynactin subunit p150 ${ }^{\text {glued }}$ (Watson et al. 2005). Overexpression of the domain of p $150^{\text {glued }}$ that binds to Sec23 inhibits the export of VSV-G from the ER, suggesting that this interaction may anchor vesicles to the dynein motor (Watson et al. 2005). Although not essential for ER-ERGIC trafficking, microtubules may stimulate the export of cargo from the ER (Watson et al. 2005). It is also possible that dynein is recruited to COPII vesicles to regulate a later microtubule-dependent step in ER-Golgi transport. TRAPPC9, a subunit of the mammalian TRAPP complex described below (Yamasaki et al. 2009), also interacts with p150 ${ }^{\text {glued }}$ (Zong et al. 2012). Likewise, retrograde traffic from the ERGIC to the ER is driven by microtubules but uses kinesin, a microtubule-plus-end-directed motor (Lippincott-Schwartz et al. 1995). The dual activities of dynein and kinesin may play a role in positioning the ERGIC on the ER-Golgi pathway. 


\section{VESICLE TETHERING}

Tethering factors link vesicles to their correct acceptor membrane. The tethering of COPII vesicles to the ERGIC/Golgi is dependent on the Sec23 subunit of the COPII coat complex, the multimeric TRAPP complex, the Rab Ypt1 (Rab1 in mammals), and the Ypt1 effector Uso1 (p115 in mammals) (Cai et al. 2007a).

Like many other vesicle delivery events, a Rab family protein coordinates the tethering and fusion of COPII vesicles. There are at least 60 Rabs in mammalian cells and 11 Ypt proteins in the yeast $S$. cerevisiae (Hutagalung and Novick 2011). These small GTPases are activated by guanine-nucleotide exchange factors (GEFs) that catalyze the exchange of GDP for GTP. The TRAPP complex acts as a GEF for Ypt1/Rab1, driving its recruitment to vesicles upon nucleotide exchange. The GEF activity of TRAPP is dependent on multiple subunits of the complex, specifically Bet3, Bet5, Trs23, and Trs31 (Kim et al. 2006). A crystal structure of these subunits bound to Ypt1 revealed that Ypt1 largely binds to the highly conserved surface of Trs23 (Cai et al. 2008). Key to TRAPP's catalytic activity is the interaction of the carboxyl terminus of Bet3 with two regions (switch I and switch II) in the nucleotide-binding pocket of the Rab. When bound to Bet3, the switch regions adopt a different conformation, which leads to the opening of the nucleotide-binding pocket. Bet5 mainly plays a structural role in the complex, facilitating the interaction of Trs23 with the carboxyl terminus of Bet3, whereas Trs31 may have an allosteric role. Together these molecular interactions catalyze the release of GDP from Ypt1. TRAPP also accelerates the uptake of GTP by holding the switch regions in an open conformation.

The activated form of the Rab (Rab-GTP) exerts its function through its effectors. One of the best-characterized Rab1 effectors is the Golgin p115. Golgins are a family of coiled-coil proteins that play a role in structuring the Golgi (Barr and Short 2003). p115 is recruited to COPII vesicles by activated Rab1 (Allan et al. 2000), where it mediates COPII vesicle tethering (Bentley et al. 2006) and appears to play a role in organizing tER sites (Kondylis and Rabouille 2003). p115 also resides on the ERGIC (Alvarez et al. 1999) and Golgi (Nelson et al. 1998). In addition to regulating ER-ERGIC traffic, p115 is required for ERGIC-Golgi transport (Alvarez et al. 1999), where it works in conjunction with another Golgin and Rab1 effector, GM130. Additional players in this event include the Golgin GRASP-65 (Moyer et al. 2001) and the COPI coat subunit $\beta$-COP, which interacts with p115 during tethering events at the Golgi (Guo et al. 2008).

Uso1, an ortholog of p115, mediates the tethering of COPII vesicles to the Golgi in yeast (Cao et al. 1998). Uso 1 and p115 share structural characteristics that are essential for their tethering functions. Electron microscopy analysis showed that both Uso1 and p115 are long coiled-coil dimers with two globular head regions, similar to the motor protein myosin (Sapperstein et al. 1995; Yamakawa et al. 1996). The amino-terminal head region of p115 contains 12 Armadillo-like $\alpha$-helical repeats, termed tether repeats (TRs), which are also found in other tethering factors (An et al. 2009). The conserved $\mathrm{H} 1 \mathrm{TR}$ is required for the interaction of p115 with Rab1-GTP (An et al. 2009). The coiledcoil region of Uso1 has an average length of $154 \mathrm{~nm}$ that can bend in defined regions (Yamakawa et al. 1996). This long length may facilitate the tethering of vesicles that are a considerable distance away from the Golgi. Bending would allow Uso1 to bring the vesicle closer to the Golgi, where fusion occurs. The first coiledcoil region of p115 (CC1) binds to the ER-Golgi SNAREs, suggesting a role in mediating fusion in addition to tethering (Shorter et al. 2002). The carboxy-terminal tail of p115 binds to two other Golgins, GM130 (Nakamura et al. 1997) and giantin (Lesa et al. 2000; Linstedt et al. 2000), which compete for binding to p 115 (Linstedt et al. 2000). p115 may initially engage the SNAREs and giantin on the vesicle before it binds to GM130 on the Golgi (Sonnichsen et al. 1998; Allan et al. 2000). In yeast, the golgin orthologs Bug1 (GM130) and Grh1 (GRASP65) interact with both Uso1 and the Sec23/Sec24 complex, suggesting that conserved interactions drive vesicle tethering. A yeast ortholog 
C. Lord et al.

for giantin has not been identified thus far (Behnia et al. 2007).

\section{VESICLE FUSION}

Once tethered, the vesicle fuses with its target membrane. Fusion is catalyzed by a class of proteins called SNAREs, which are generally type II membrane proteins that are anchored via a carboxy-terminal transmembrane domain or a hydrophobic posttranslational modification (Veit et al. 1996; McNew et al. 1997). They bind to each other through a conserved 60- to 70-amino-acid heptad repeat, called the SNARE motif (Fasshauer et al. 1998; Weber et al. 1998). SNAREs are divided into four subgroups: R-, Qa-, Qb-, and Qc-SNAREs. This subgrouping is based on the SNARE domain sequence and the presence of a glutamine or asparagine at a conserved site in the protein (Fasshauer et al. 1998; Bock et al. 2001). In vivo (Newman et al. 1990) and in vitro (Lian and Ferro-Novick 1993; Cao and Barlowe 2000) studies suggest that the ER-Golgi SNAREs Sec22 (R), Sed5 $(\mathrm{Qa}), \operatorname{Bos} 1(\mathrm{Qb})$, and Bet1 $(\mathrm{Qc})$ regulate fusion in yeast. Their orthologs-Sec22, syntaxin 5 (Sed5), membrin (Bos1), and Bet1-appear to act in both ER-ERGIC and ERGIC-Golgi traffic (Hay et al. 1997, 1998; Bentley et al. 2006). ER-ERGIC traffic may use a long isoform of syntaxin-5 (Hay et al. 1997; Hui et al. 1997). A second SNARE complex-syntaxin-5, Bet1, GOS-28, and Ykt6 - also functions in the late stages of ER-Golgi traffic in mammalian cells (Zhang and Hong 2001).

The localization and structure of SNAREs are critical for their function in membrane fusion. In vitro liposome studies suggest that fusion occurs when Bos1, Sec22, and Sed5 are topologically restricted from Bet1 (Parlati et al. 2000). Fusion requires the formation of a trans-SNARE complex, which occurs when the SNARE motifs on opposing membranes bind to each other to form a highly stable parallel helical bundle (Sutton et al. 1998). Formation of this bundle proceeds like a zipper (Lin and Scheller 1997), starting at the amino terminus of each SNARE. Zippering is thought to provide the mechanical force necessary to overcome the significant energy barrier required to fuse two membranes together. Once fusion is completed, the SNAREs remain associated in a cis-SNARE complex at the target membrane. This cisSNARE complex is disassembled by SNAP/ Sec17 and the $\mathrm{AAA}^{+}$ATPase NSF/Sec18 in an ATP-dependent manner. Disassembly is essential to reprime the SNAREs for further rounds of membrane fusion (Mayer et al. 1996).

Other factors associated with the SNAREs regulate membrane fusion. For example, p115 not only binds to the SNAREs (Allan et al. 2000), but it also catalyzes SNARE complex formation (Shorter et al. 2002). Sly1, a Sec1/ Munc18 (SM) family member that binds the Qa-SNARE syntaxin 5/Sed5p (Dascher and Balch 1996), is required for ER-Golgi transport (Cao and Barlowe 2000; Williams et al. 2004) and has been implicated in SNARE pairing (Flanagan and Barlowe 2006). Its precise role in membrane fusion, however, is still unclear. Sly1 may increase the rate of SNARE pairing (Kosodo et al. 2002); however, another study found it primarily prevents the ER-Golgi SNAREs from pairing promiscuously with other SNAREs acting at different stages of membrane traffic (Peng and Gallwitz 2002). This latter study suggested that Sly1 may only function to promote the specific binding of Sed5 with Bet1, Bos1, and Sec22.

\section{THE DIRECTIONALITY OF ER-GOLGI TRAFFIC IS MEDIATED BY THE COPII COAT}

The COPII coat subunit Sec23 initiates vesicle tethering by recruiting Bet3 to COPII-coated vesicles (Yu et al. 2006; Cai et al. 2007b). Bet3 is a component of the multimeric TRAPP complex, described above, which activates Rab1 and its yeast ortholog Ypt1 (Cai et al. 2008; Yamasaki et al. 2009). Interestingly, the recruitment of Bet 3 to vesicles is dependent on the release of Sar1-GTP from the vesicle (Lord et al. 2011). Because the hydrolysis of GTP on Sarl is required for vesicle fission (Bielli et al. 2005; Lee et al. 2005), these events ensure that tethering is only initiated after vesicle budding takes place. After Sar1 is released from the membrane, the coat is retained, presumably by cargo sorted 
COPII Coat Complex

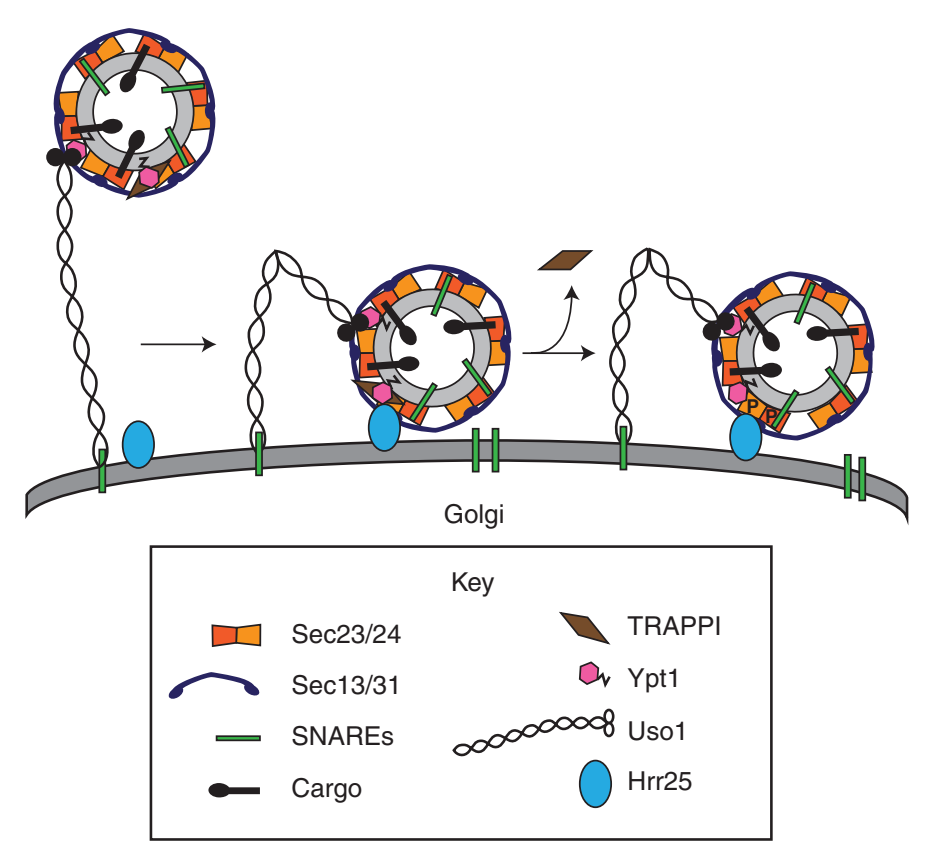

Figure 3. Vesicle tethering and fusion is regulated by the COPII coat complex. The TRAPPI complex is recruited to COPII vesicles by the coat subunit Sec23, where it activates Ypt1. On the vesicle, activated Ypt1 binds to its effector Uso1. Uso1 is a long coiled-coil tether that can bend. When the tether bends, it may bring the vesicle closer to its target. At the target membrane, the TRAPPI complex is displaced from the vesicle by the serine/ threonine kinase Hrr25/CK1 $\delta$. Hrr25/CK1 $\delta$ then phosphorylates the Sec23/Sec24 complex, the inner shell of the COPII coat. Subsequently, the coat is released, allowing the SNAREs to pair, which catalyzes membrane fusion. The vesicle does not uncoat until it reaches its acceptor compartment, ensuring that fusion only takes place at the correct membrane. These events appear to be conserved in mammalian cells.

into the vesicle (Sato and Nakano 2005; Forster et al. 2006; Cai et al. 2007b).

Activation of the Rab leads to the recruitment of the long coiled-coil tether Usol to the vesicle (Fig. 3) (Allan et al. 2000), physically linking the vesicle to its target. Long tethers, like Usol, can bend at defined regions in the coiled-coil domain (Yamakawa et al. 1996), which could bring the vesicle closer to its acceptor compartment to facilitate membrane fusion. Consistent with its role in vesicle tethering, the COPII coat remains associated with the vesicle until tethering is complete (Lord et al. 2011). At the Golgi, Hrr25 (the yeast homolog of CK1 $\delta$ ), a serine/threonine kinase, could displace TRAPPI from the COPII coat and phosphorylate the Sec23/Sec24 complex. These events ensure the fidelity of membrane traffic by preventing the vesicle from fusing prema- turely (Lord et al. 2011). Together, these findings imply that phosphorylation of the COPII coat regulates COPII vesicle fusion.

\section{CONCLUDING REMARKS}

Genetic, biochemical, and live cell imaging studies have revealed that the basic mechanism of ER-Golgi traffic is conserved from yeast to man. Despite a detailed understanding of the mechanisms that drive this process, our understanding of how the pathway is regulated and integrated to maintain organelle homeostasis remains murky. An in-depth picture of how cargo loading, vesicle budding, tethering, and fusion are coordinated and the role that posttranslational modifications play in these events still needs to be elucidated, and many questions remain unanswered. For example, we still do not 


\section{Lord et al.}

fully understand how GTPase hydrolysis is coupled to cargo loading and the role that Sec16 plays in these events. Ubiquitination is clearly playing an important role in loading large cargo, but are other posttranslational modifications also involved? Phosphorylation by a Golgi-localized kinase is required for membrane fusion, but its precise role is still unknown. It is also unknown if other kinases are required for fusion and the role that phosphatases play in ER-Golgi trafficking. Interestingly, a recent report has identified several kinases and phosphatases that regulate secretion in mammalian cells (Farhan et al. 2010). Now that we have identified the players and the posttranslational modifications that may regulate these processes, we have set the stage to answer these important questions.

\section{ACKNOWLEDGMENTS}

E.A.M. is supported by National Institutes of Health grants R01-GM085089 and R01GM078186. S.F.-N. is an Investigator of the Howard Hughes Medical Institute.

\section{REFERENCES}

* Reference is also in this collection.

Allan BB, Moyer BD, Balch WE. 2000. Rab1 recruitment of p115 into a cis-SNARE complex: Programming budding COPII vesicles for fusion. Science 289: 444-448.

Alvarez C, Fujita H, Hubbard A, Sztul E. 1999. ER to Golgi transport: Requirement for p115 at a pre-Golgi VTC stage. J Cell Biol 147: 1205-1222.

An Y, Chen CY, Moyer B, Rotkiewicz P, Elsliger MA, Godzik A, Wilson IA, Balch WE. 2009. Structural and functional analysis of the globular head domain of p115 provides insight into membrane tethering. $J \mathrm{Mol}$ Biol 391: 26-41.

Antonny B, Madden D, Hamamoto S, Orci L, Schekman R. 2001. Dynamics of the COPII coat with GTP and stable analogues. Nat Cell Biol 3: 531-537.

Aridor M, Bannykh SI, Rowe T, Balch WE. 1995. Sequential coupling between COPII and COPI vesicle coats in endoplasmic reticulum to Golgi transport. J Cell Biol 131: 875-893.

Aridor M, Weissman J, Bannykh S, Nuoffer C, Balch WE. 1998. Cargo selection by the COPII budding machinery during export from the ER. J Cell Biol 141: 61-70.

Baker D, Hicke L, Rexach M, Schleyer M, Schekman R. 1988. Reconstitution of SEC gene product-dependent intercompartmental protein transport. Cell 54: 335-344.
Bannykh SI, Rowe T, Balch WE. 1996. The organization of endoplasmic reticulum export complexes. J Cell Biol 135: 19-35.

Barlowe C, Schekman R. 1993. SEC12 encodes a guaninenucleotide-exchange factor essential for transport vesicle budding from the ER. Nature 365: 347-349.

Barlowe C, d'Enfert C, Schekman R. 1993. Purification and characterization of SAR1p, a small GTP-binding protein required for transport vesicle formation from the endoplasmic reticulum. J Biol Chem 268: 873-879.

Barlowe C, Orci L, Yeung T, Hosobuchi M, Hamamoto S, Salama N, Rexach MF, Ravazzola M, Amherdt M, Schekman R. 1994. COPII: A membrane coat formed by Sec proteins that drive vesicle budding from the endoplasmic reticulum. Cell 77: 895-907.

Barr FA, Short B. 2003. Golgins in the structure and dynamics of the Golgi apparatus. Curr Opin Cell Biol 15: 405413.

Behnia R, Barr FA, Flanagan JJ, Barlowe C, Munro S. 2007. The yeast orthologue of GRASP65 forms a complex with a coiled-coil protein that contributes to ER to Golgi traffic. J Cell Biol 176: 255-261.

Ben-Tekaya H, Miura K, Pepperkok R, Hauri HP. 2005. Live imaging of bidirectional traffic from the ERGIC. J Cell Sci 118: $357-367$.

Bentley M, Liang Y, Mullen K, Xu D, Sztul E, Hay JC. 2006. SNARE status regulates tether recruitment and function in homotypic COPII vesicle fusion. J Biol Chem 281: 38825-38833.

Bi X, Corpina RA, Goldberg J. 2002. Structure of the Sec23/ 24-Sar1 pre-budding complex of the COPII vesicle coat. Nature 419: 271-277.

Bi X, Mancias JD, Goldberg J. 2007. Insights into COPII coat nucleation from the structure of Sec23.Sar1 complexed with the active fragment of Sec31. Dev Cell 13: 635-645.

Bielli A, Haney CJ, Gabreski G, Watkins SC, Bannykh SI, Aridor M. 2005. Regulation of Sar1 NH2 terminus by GTP binding and hydrolysis promotes membrane deformation to control COPII vesicle fission. J Cell Biol 171: 919-924.

Bock J, Matern H, Peden A, Scheller R. 2001. A genomic perspective on membrane compartment organization. Nature 409: 839-841.

Cai H, Reinisch K, Ferro-Novick S. 2007a. Coats, tethers, Rabs, and SNAREs work together to mediate the intracellular destination of a transport vesicle. Dev Cell 12: 671-682.

Cai H, Yu S, Menon S, Cai Y, Lazarova D, Fu C, Reinisch K, Hay JC, Ferro-Novick S. 2007b. TRAPPI tethers COPII vesicles by binding the coat subunit Sec23. Nature 445: 941-944.

Cai Y, Chin HF, Lazarova D, Menon S, Fu C, Cai H, Sclafani A, Rodgers DW, De La Cruz EM, Ferro-Novick S, et al. 2008. The structural basis for activation of the Rab Yptlp by the TRAPP membrane-tethering complexes. Cell 133: 1202-1213.

Cao X, Barlowe C. 2000. Asymmetric requirements for a Rab GTPase and SNARE proteins in fusion of COPII vesicles with acceptor membranes. J Cell Biol 149: 55-66. 
Cao X, Ballew N, Barlowe C. 1998. Initial docking of ERderived vesicles requires Usolp and Yptlp but is independent of SNARE proteins. EMBO J 17: 2156-2165.

Copic A, Latham CF, Horlbeck MA, D’Arcangelo JG, Miller EA. 2012. ER cargo properties specify a requirement for COPII coat rigidity mediated by Sec13p. Science 335: $1359-1362$

Dascher C, Balch WE. 1996. Mammalian Sly1 regulates syntaxin 5 function in endoplasmic reticulum to Golgi transport. J Biol Chem 271: 15866-15869.

D'Souza-Schorey C, Chavrier P. 2006. ARF proteins: Roles in membrane traffic and beyond. Nat Rev Mol Cell Biol 7: 347-358.

Farhan H, Reiterer V, Kriz A, Hauri H-P, Pavelka M, Sitte HH, Freissmuth M. 2008. Signal-dependent export of GABA transporter 1 from the ER-Golgi intermediate compartment is specified by a C-terminal motif. J Cell Sci 121: $753-761$.

Farhan H, Wendeler MW, Mitrovic S, Fava E, Silberberg Y, Sharan R, Zerial M, Hauri H-P. 2010. MAPK signaling to the early secretory pathway revealed by kinase/phosphatase functional screening. J Cell Biol 189: 997-1011.

Fasshauer D, Eliason WK, Brunger AT, Jahn R. 1998. Identification of a minimal core of the synaptic SNARE complex sufficient for reversible assembly and disassembly. Biochemistry 37: 10354-10362.

Flanagan JJ, Barlowe C. 2006. Cysteine-disulfide cross-linking to monitor SNARE complex assembly during endoplasmic reticulum-Golgi transport. J Biol Chem 281: 2281-2288.

Forster R, Weiss M, Zimmermann T, Reynaud EG, Verissimo F, Stephens DJ, Pepperkok R. 2006. Secretory cargo regulates the turnover of COPII subunits at single ER exit sites. Curr Biol 16: 173-179.

Fromme JC, Ravazzola M, Hamamoto S, Al-Balwi M, Eyaid W, Boyadjiev SA, Cosson P, Schekman R, Orci L. 2007. The genetic basis of a craniofacial disease provides insight into COPII coat assembly. Dev Cell 13: 623-634.

Giraudo CG, Maccioni HJF. 2003. Endoplasmic reticulum export of glycosyltransferases depends on interaction of a cytoplasmic dibasic motif with Sar1. Mol Biol Cell 14: 3753-3766.

Guo Y, Punj V, Sengupta D, Linstedt AD. 2008. Coat-tether interaction in Golgi organization. Mol Biol Cell 19: 2830-2843.

Hay JC, Chao DS, Kuo CS, Scheller RH. 1997. Protein interactions regulating vesicle transport between the endoplasmic reticulum and Golgi apparatus in mammalian cells. Cell 89: 149-158.

Hay JC, Klumperman J, Oorschot V, Steegmaier M, Kuo CS, Scheller RH. 1998. Localization, dynamics, and protein interactions reveal distinct roles for ER and Golgi SNAREs. J Cell Biol 141: 1489-1502.

Hsu VW, Lee SY, Yang JS. 2009. The evolving understanding of COPI vesicle formation. Nat Rev Mol Cell Biol 10: 360-364.

Hughes H, Budnik A, Schmidt K, Palmer KJ, Mantell J, Noakes C, Johnson A, Carter DA, Verkade P, Watson P, et al. 2009. Organisation of human ER-exit sites: Requirements for the localisation of Sec16 to transitional ER. J Cell Sci 122: 2924-2934.
Huh W, Falvo J, Gerke L, Carroll A, Howson R, Weissman J, O'Shea E. 2003. Global analysis of protein localization in budding yeast. Nature 425: 686-691.

Hui N, Nakamura N, Sönnichsen B, Shima DT, Nilsson T, Warren G. 1997. An isoform of the Golgi t-SNARE, syntaxin 5, with an endoplasmic reticulum retrieval signal. Mol Biol Cell 8: 1777-1787.

Hutagalung AH, Novick PJ. 2011. Role of Rab GTPases in membrane traffic and cell physiology. Physiol Rev 91: 119-149.

Ivan V, de Voer G, Xanthakis D, Spoorendonk KM, Kondylis V, Rabouille C. 2008. Drosophila Sec16 mediates the biogenesis of tER sites upstream of Sar1 through an arginine-rich motif. Mol Biol Cell 19: 4352-4365.

Jackson MR, Nilsson T, Peterson PA. 1990. Identification of a consensus motif for retention of transmembrane proteins in the endoplasmic reticulum. EMBO J 9: $3153-$ 3162.

Jin L, Pahuja KB, Wickliffe KE, Gorur A, Baumgartel C, Schekman R, Rape M. 2012. Ubiquitin-dependent regulation of COPII coat size and function. Nature 482: 495-500.

Kardon JR, Vale RD. 2009. Regulators of the cytoplasmic dynein motor. Nat Rev Mol Cell Biol 10: 854-865.

Kim Y, Raunser S, Munger C, Wagner J, Song Y, Cygler M, Walz T, Oh B, Sacher M. 2006. The architecture of the multisubunit TRAPP I complex suggests a model for vesicle tethering. Cell 127: 817-830.

Kondylis V, Rabouille C. 2003. A novel role for dp115 in the organization of tER sites in Drosophila. J Cell Biol 162: 185-198.

Kosodo Y, Noda Y, Adachi H, Yoda K. 2002. Binding of Sly1 to Sed5 enhances formation of the yeast early Golgi SNARE complex. J Cell Sci 115: 3683-3691.

Kuehn MJ, Herrmann JM, Schekman R. 1998. COPII-cargo interactions direct protein sorting into ER-derived transport vesicles. Nature 391: 187-190.

Kung LF, Pagant S, Futai E, D’Arcangelo JG, Buchanan R, Dittmar JC, Reid RJ, Rothstein R, Hamamoto S, Snapp EL, et al. 2012. Sec24p and Sec16p cooperate to regulate the GTP cycle of the COPII coat. EMBO J 31: $1014-$ 1027.

Lee MCS, Hamamoto S, Schekman R. 2002. Ceramide biosynthesis is required for the formation of the oligomeric $\mathrm{H}^{+}$-ATPase Pmalp in the yeast endoplasmic reticulum. $J$ Biol Chem 277: 22395-22401.

Lee MCS, Orci L, Hamamoto S, Futai E, Ravazzola M, Schekman R. 2005. Sarlp N-terminal helix initiates membrane curvature and completes the fission of a COPII vesicle. Cell 122: 605-617.

Lesa GM, Seemann J, Shorter J, Vandekerckhove J, Warren G. 2000. The amino-terminal domain of the Golgi protein giantin interacts directly with the vesicle-tethering protein p115. J Biol Chem 275: 2831-2836.

Letourneur F, Gaynor E, Hennecke S, Demolliere C, Duden R, Emr S, Riezman H, Cosson P. 1994. Coatomer is essential for retrieval of dilysine-tagged proteins to the endoplasmic reticulum. Cell 79: 1199-1207.

Lian JP, Ferro-Novick S. 1993. Boslp, an integral membrane protein of the endoplasmic reticulum to Golgi transport 
C. Lord et al.

vesicles, is required for their fusion competence. Cell 73: $735-745$.

Lin RC, Scheller RH. 1997. Structural organization of the synaptic exocytosis core complex. Neuron 19: 10871094.

Linstedt AD, Jesch SA, Mehta A, Lee TH, Garcia-Mata R, Nelson DS, Sztul E. 2000. Binding relationships of membrane tethering components. The giantin $\mathrm{N}$ terminus and the GM130 N terminus compete for binding to the p115 C terminus. J Biol Chem 275: 10196-10201.

Lippincott-Schwartz J, Cole NB, Marotta A, Conrad PA, Bloom GS. 1995. Kinesin is the motor for microtubulemediated Golgi-to-ER membrane traffic. J Cell Biol 128: 293-306.

Lord C, Bhandari D, Menon S, Ghassemian M, Nycz D, Hay J, Ghosh P, Ferro-Novick S. 2011. Sequential interactions with Sec23 control the direction of vesicle traffic. Nature 473: 181-186.

Malhotra V, Erlmann P. 2011. Protein export at the ER: Loading big collagens into COPII carriers. EMBO J 30: 3475-3480.

Mancias JD, Goldberg J. 2007. The transport signal on Sec22 for packaging into COPII-coated vesicles is a conformational epitope. Mol Cell 26: 403-414.

Mancias JD, Goldberg J. 2008. Structural basis of cargo membrane protein discrimination by the human COPII coat machinery. EMBO J 27: 2918-2928.

Martínez-Menárguez JA, Geuze HJ, Slot JW, Klumperman J. 1999. Vesicular tubular clusters between the ER and Golgi mediate concentration of soluble secretory proteins by exclusion from COPI-coated vesicles. Cell 98: 81-90.

Matsuoka K, Morimitsu Y, Uchida K, Schekman R. 1998a. Coat assembly directs v-SNARE concentration into synthetic COPII vesicles. Mol Cell 2: 703-708.

Matsuoka K, Orci L, Amherdt M, Bednarek SY, Hamamoto S, Schekman R, Yeung T. 1998b. COPII-coated vesicle formation reconstituted with purified coat proteins and chemically defined liposomes. Cell 93: 263-275.

Mayer A, Wickner W, Haas A. 1996. Sec18p (NSF)-driven release of Sec17p ( $\alpha$-SNAP) can precede docking and fusion of yeast vacuoles. Cell 85: 83-94.

McNew JA, Sogaard M, Lampen NM, Machida S, Ye RR, Lacomis L, Tempst P, Rothman JE, Sollner TH. 1997. Ykt6p, a prenylated SNARE essential for endoplasmic reticulum-Golgi transport. J Biol Chem 272: $17776-$ 17783.

Mezzacasa A, Helenius A. 2002. The transitional ER defines a boundary for quality control in the secretion of tsO45 VSV glycoprotein. Traffic 3: 833-849.

Miller E, Antonny B, Hamamoto S, Schekman R. 2002. Cargo selection into COPII vesicles is driven by the Sec24p subunit. EMBO J 21: 6105-6113.

Miller EA, Beilharz TH, Malkus PN, Lee MCS, Hamamoto S, Orci L, Schekman R. 2003. Multiple cargo binding sites on the COPII subunit Sec24p ensure capture of diverse membrane proteins into transport vesicles. Cell 114: 497-509.

Mossessova E, Bickford LC, Goldberg J. 2003. SNARE selectivity of the COPII coat. Cell 114: 483-495.
Moyer BD, Allan BB, Balch WE. 2001. Rab1 interaction with a GM130 effector complex regulates COPII vesicle cisGolgi tethering. Traffic 2: 268-276.

Muniz M, Morsomme P, Riezman H. 2001. Protein sorting upon exit from the endoplasmic reticulum. Cell 104: 313-320.

Munro S, Pelham HR. 1987. A C-terminal signal prevents secretion of luminal ER proteins. Cell 48: 899-907.

Nakamura N, Lowe M, Levine TP, Rabouille C, Warren G. 1997. The vesicle docking protein p115 binds GM130, a cis-Golgi matrix protein, in a mitotically regulated manner. Cell 89: 445-455.

Nakano A, Muramatsu M. 1989. A novel GTP-binding protein, Sarlp, is involved in transport from the endoplasmic reticulum to the Golgi apparatus. J Cell Biol 109: 26772691.

Nakano A, Brada D, Schekman R. 1988. A membrane glycoprotein, Sec12p, required for protein transport from the endoplasmic reticulum to the Golgi apparatus in yeast. J Cell Biol 107: 851-863.

Nelson DS, Alvarez C, Gao YS, Garcia-Mata R, Fialkowski E, Sztul E. 1998. The membrane transport factor TAP/p115 cycles between the Golgi and earlier secretory compartments and contains distinct domains required for its localization and function. J Cell Biol 143: 319-331.

Newman AP, Shim J, Ferro-Novick S. 1990. BET1, BOS1, and SEC22 are members of a group of interacting yeast genes required for transport from the endoplasmic reticulum to the Golgi complex. Mol Cell Biol 10: 3405-3414.

Nichols WC, Ginsburg D. 1999. From the ER to the Golgi: Insights from the study of combined factors V and VIII deficiency. Am J Hum Genet 64: 1493-1498.

Novick P, Field C, Schekman R. 1980. Identification of 23 complementation groups required for post-translational events in the yeast secretory pathway. Cell 21: 205-215.

Novick P, Ferro S, Schekman R. 1981. Order of events in the yeast secretory pathway. Cell 25: 461-469.

Palade G. 1975. Intracellular aspects of the process of protein synthesis. Science 189: 347-358.

Parlati F, McNew J, Fukuda R, Miller R, Sollner T, Rothman J. 2000. Topological restriction of SNARE-dependent membrane fusion. Nature 407: 194-198.

Peng R, Gallwitz D. 2002. Sly1 protein bound to Golgi syntaxin Sed5p allows assembly and contributes to specificity of SNARE fusion complexes. J Cell Biol 157: 645-655.

Pepperkok R, Scheel J, Horstmann H, Hauri HP, Griffiths G, Kreis TE. 1993. $\beta$-COP is essential for biosynthetic membrane transport from the endoplasmic reticulum to the Golgi complex in vivo. Cell 74: 71-82.

Presley J, Cole N, Schroer T, Hirschberg K, Zaal K, Lippincott-Schwartz J. 1997. ER-to-Golgi transport visualized in living cells. Nature 389: 81-85.

Pucadyil TJ, Schmid SL. 2009. Conserved functions of membrane active GTPases in coated vesicle formation. Science 325: 1217-1220.

Roberg KJ, Crotwell M, Espenshade P, Gimeno R, Kaiser CA. 1999. LST1 is a SEC24 homologue used for selective export of the plasma membrane ATPase from the endoplasmic reticulum. J Cell Biol 145: 659-672.

Ruohola H, Kabcenell AK, Ferro-Novick S. 1988. Reconstitution of protein transport from the endoplasmic 
reticulum to the Golgi complex in yeast: The acceptor Golgi compartment is defective in the sec23mutant. $J$ Cell Biol 107: 1465-1476.

Saito K, Chen M, Bard F, Chen S, Zhou H, Woodley D, Polischuk R, Schekman R, Malhotra V. 2009. TANGO1 facilitates cargo loading at endoplasmic reticulum exit sites. Cell 136: 891-902.

Sapperstein S, Walter D, Grosvenor A, Heuser J, Waters M. 1995. p115 is a general vesicular transport factor related to the yeast endoplasmic reticulum to Golgi transport factor Uso1p. Proc Natl Acad Sci 92: 522-526.

Sato K, Nakano A. 2005. Dissection of COPII subunit-cargo assembly and disassembly kinetics during Sarlp-GTP hydrolysis. Nat Struct Mol Biol 12: 167-174.

Scales SJ, Pepperkok R, Kreis TE. 1997. Visualization of ERto-Golgi transport in living cells reveals a sequential mode of action for COPII and COPI. Cell 90: 1137-1148.

Schweizer A, Matter K, Ketcham CM, Hauri HP. 1991. The isolated ER-Golgi intermediate compartment exhibits properties that are different from ER and cis-Golgi. $J$ Cell Biol 113: 45-54.

Shimoni Y, Kurihara T, Ravazzola M, Amherdt M, Orci L, Schekman R. 2000. Lstlp and Sec24p cooperate in sorting of the plasma membrane ATPase into COPII vesicles in Saccharomyces cerevisiae. J Cell Biol 151: 973-984.

Shorter J, Beard M, Seemann J, Dirac-Svejstrup A, Warren G. 2002. Sequential tethering of Golgins and catalysis of SNAREpin assembly by the vesicle-tethering protein 115 . J Cell Biol 157: 45-62.

Sonnichsen B, Lowe M, Levine T, Jamsa E, Dirac-Svejstrup B, Warren G. 1998. A role for giantin in docking COPI vesicles to Golgi membranes. J Cell Biol 140: 1013-1021.

* Spang A. 2013. Retrograde traffic from the Golgi to the endoplasmic reticulum. Cold Spring Harb Perspect Biol doi: 10.1101/cshperspect.a013391.

Springer S, Schekman R. 1998. Nucleation of COPII vesicular coat complex by endoplasmic reticulum to Golgi vesicle SNAREs. Science 281: 698-700.

Stagg SM, Gürkan C, Fowler DM, LaPointe P, Foss TR, Potter CS, Carragher B, Balch WE. 2006. Structure of the Sec13/31 COPII coat cage. Nature 439: 234-238.

Stagg SM, LaPointe P, Razvi A, Gürkan C, Potter CS, Carragher B, Balch WE. 2008. Structural basis for cargo regulation of COPII coat assembly. Cell 134: 474-484.

Stephens DJ, Pepperkok R. 2002. Imaging of procollagen transport reveals COPI-dependent cargo sorting during ER-to-Golgi transport in mammalian cells. J Cell Sci 115: $1149-1160$.

Sutton RB, Fasshauer D, Jahn R, Brunger AT. 1998. Crystal structure of a SNARE complex involved in synaptic exocytosis at $2.4 \AA$ resolution. Nature 395: 347-353.
Tabata K, Sato K, Ide T, Nishizaka T, Nakano A, Noji H. 2009. Visualization of cargo concentration by COPII minimal machinery in a planar lipid membrane. $E M B O$ J 28: 3279-3289.

Townley AK, Feng Y, Schmidt K, Carter DA, Porter R, Verkade P, Stephens DJ. 2008. Efficient coupling of Sec23-Sec24 to Sec13-Sec31 drives COPII-dependent collagen secretion and is essential for normal craniofacial development. J Cell Sci 121: 3025-3034.

Veit M, Sollner TH, Rothman JE. 1996. Multiple palmitoylation of synaptotagmin and the t-SNARE SNAP-25. FEBS Lett 385: 119-123.

Watanabe R, Riezman H. 2004. Differential ER exit in yeast and mammalian cells. Curr Opin Cell Biol 16: 350-355.

Watson P, Forster R, Palmer KJ, Pepperkok R, Stephens DJ. 2005. Coupling of ER exit to microtubules through direct interaction of COPII with dynactin. Nat Cell Biol 7: $48-55$.

Weber T, Zemelman BV, McNew JA, Westermann B, Gmachl M, Parlati F, Sollner TH, Rothman JE. 1998. SNAREpins: Minimal machinery for membrane fusion. Cell 92: 759-772.

Williams AL, Ehm S, Jacobson NC, Xu D, Hay JC. 2004. rsly1 binding to syntaxin 5 is required for endoplasmic reticulum-to-Golgi transport but does not promote SNARE motif accessibility. Mol Biol Cell 15: 162-175.

Witte K, Schuh AL, Hegermann J, Sarkeshik A, Mayers JR, Schwarze K, Yates JR, Eimer S, Audhya A. 2011. Mechanisms by which TFG functions in protein secretion and oncogenesis. Nat Cell Biol 13: 550-558.

Yamakawa H, Seog D, Yoda K, Yamasaki M, Wakabayashi T. 1996. Usol protein is a dimer with two globular heads and a long coiled-coil tail. J Struct Biol 116: 356-365.

Yamasaki A, Menon S, Yu S, Barrowman J, Meerloo T, Oorschot V, Klumperman J, Satoh A, Ferro-Novick S. 2009. mTrs130 is a component of a mammalian TRAPPII complex, a Rab1 GEF that binds to COPI-coated vesicles. Mol Biol Cell 20: 4205-4215.

Yoshihisa T, Barlowe C, Schekman R. 1993. Requirement for a GTPase-activating protein in vesicle budding from the endoplasmic reticulum. Science 259: 1466-1468.

Yu S, Satoh A, Pypaert M, Mullen K, Hay JC, Ferro-Novick S. 2006. mBet3p is required for homotypic COPII vesicle tethering in mammalian cells. J Cell Biol 174: 359-368.

Zhang T, Hong W. 2001. Ykt6 forms a SNARE complex with syntaxin 5, GS28, and Bet1 and participates in a late stage in endoplasmic reticulum-Golgi transport. J Biol Chem 276: $27480-27487$.

Zong M, Satoh A, Yu MK, Siu KY, Ng WY, Chan HC, Tanner JA, Yu S. 2012. TRAPPC9 mediates the interaction between p150 and COPII vesicles at the target membrane. PLOS ONE 7: e29995. 


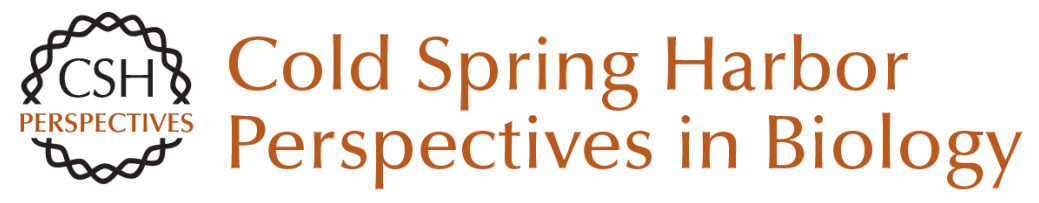

\title{
The Highly Conserved COPII Coat Complex Sorts Cargo from the Endoplasmic Reticulum and Targets It to the Golgi
}

\author{
Christopher Lord, Susan Ferro-Novick and Elizabeth A. Miller
}

Cold Spring Harb Perspect Biol 2013; doi: 10.1101/cshperspect.a013367

Subject Collection The Endoplasmic Reticulum

Sorting and Export of Proteins at the Endoplasmic

Reticulum Ishier Raote, Sonashree Saxena and Vivek Malhotra

Endoplasmic Reticulum Membrane Contact Sites, Lipid Transport, and Neurodegeneration Andrés Guillén-Samander and Pietro De Camilli

AMPylation and Endoplasmic Reticulum Protein Folding Homeostasis Luke A. Perera and David Ron

The Endoplasmic Reticulum and the Fidelity of Nascent Protein Localization

Michael J. McKenna and Sichen Shao

Endoplasmic Reticulum Architecture and Inter-Organelle Communication in Metabolic Health and Disease

Ana Paula Arruda and Günes Parlakgül

Regulation and Functions of the ER-Associated Nrf1 Transcription Factor

Gary Ruvkun and Nicolas Lehrbach

Mechanism of Protein Translocation by the Sec61 Translocon Complex

Samuel Itskanov and Eunyong Park
Glycerolipid Synthesis and Lipid Droplet

Formation in the Endoplasmic Reticulum Robert V. Farese, Jr. and Tobias C. Walther

The Biogenesis of Multipass Membrane Proteins Luka Smalinskaite and Ramanujan S. Hegde

A TAle of Two Pathways: Tail-Anchored Protein Insertion at the Endoplasmic Reticulum Alina Guna, Masami Hazu, Giovani Pinton Tomaleri, et al.

Cholesterol Transport to the Endoplasmic Reticulum John P. Kennelly and Peter Tontonoz

The Role of the Rhomboid Superfamily in ER Protein Quality Control: From Mechanisms and Functions to Diseases

Satarupa Bhaduri, Nicola A. Scott and Sonya E. Neal

ER-Phagy: Quality and Quantity Control of the

Endoplasmic Reticulum by Autophagy Haruka Chino and Noboru Mizushima

Structure and Function of the Nuclear Pore

Complex

Stefan Petrovic, George W. Mobbs, Christopher J. Bley, et al.

For additional articles in this collection, see http://cshperspectives.cshlp.org/cgi/collection/

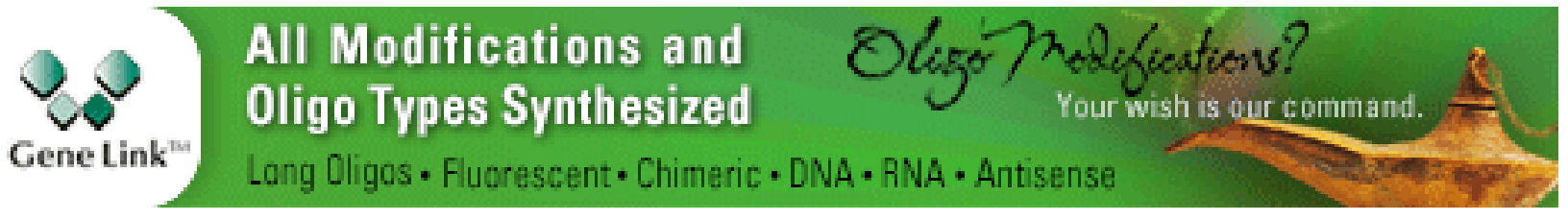




\section{Evolutionary Aspects of the Unfolded Protein Response \\ Kazutoshi Mori}

Post-Translational Regulation of HMG CoA Reductase

Youngah Jo and Russell A. DeBose-Boyd

For additional articles in this collection, see http://cshperspectives.cshlp.org/cgi/collection/

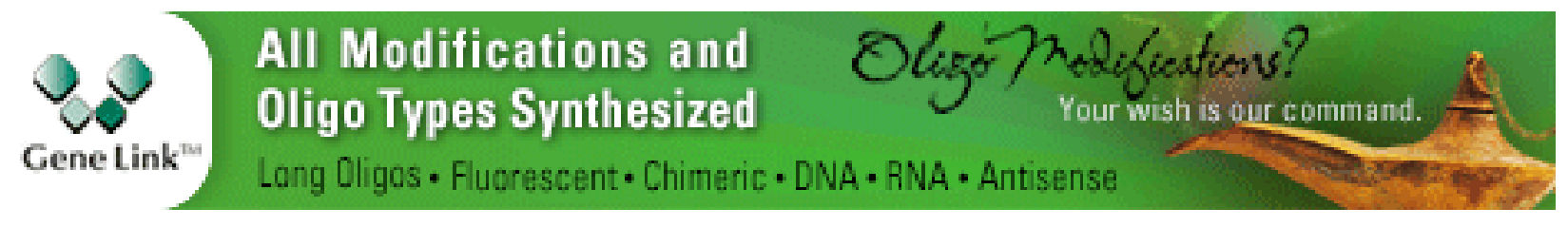

Copyright @ 2013 Cold Spring Harbor Laboratory Press; all rights reserved 